\title{
Modelling Observational Constraints for Dark Matter Halos
}

\author{
F.D.A. Hartwick \\ Department of Physics and Astronomy, \\ University of Victoria, Victoria, BC, Canada, V8W $3 P 6$
}

\begin{abstract}
Observations show that the underlying rotation curves at intermediate radii in spiral and low-surface brightness galaxies are nearly universal. Further, in these same galaxies, the product of the central density and the core radius $\left(\rho_{0} r_{0}\right)$ is constant. An empirically motivated model for dark matter halos which incorporates these observational constraints is presented and shown to be in accord with the observations. A model fit to the observations of the galaxy cluster Abell 611 shows that $\rho_{0} r_{0}$ for the dark matter halo in this more massive structure is larger by a factor of $\sim 20$ over that assumed for the galaxies. The model maintains the successful NFW form in the outer regions although the well defined differences in the inner regions suggest that modifications to the standard CDM picture are required.
\end{abstract}

Subject headings: cosmology: dark matter

\section{Introduction}

Recent observations suggest that many spiral galaxies possess constant density cores whose central densities are related to their core radii. In addition these same galaxies possess nearly universal rotation curves at intermediate radii after removal of the baryonic component. These observations are used to construct a simple empirical model for the density distribution of dark matter halos. This is done without prejudice for any specific model of structure formation although the model is designed to retain the sucessful NFW form (Navarro et al. 1997) in the outer regions.

For many years we have relied on sophisticated cosmological simulations to provide guidance on the distribution of dark matter (hence the NFW profile). Only relatively recently has observational data on the distribution of dark matter over a large range in radius become available (e.g. the data on the galaxy cluster Abell 611 from Newman et al. 2009). If the empirical model derived from the galaxy sample above is to describe all dark matter halos 
and hence to provide constraints on the properties of the dark matter particle itself then it is important to test it on as many scales and morphologies as possible. For this reason we include an application of our model to the observations of the galaxy cluster Abell 611 .

\section{The Model}

The model is motivated by two independent observational results. The first is from Donato et al. (2009) who showed that the product of the central dark matter density and its core radius (the radius within which the density remains approximately constant) is a constant with a value of $\sim 120 M_{\odot} p c^{-2}$. Salucci et al. (2011) emphasize that this result extends to the dwarf spheroidal galaxies as well. This result builds upon earlier work by Kormendy \& Freeman (2004). The second observational result is from the work of McGaugh et al. (2007). Here an apparently universal relation between rotational velocity and radius at intermediate radii in 60 spiral galaxies is found after removal of the contributions from the stars and gas. Walker et al. (2010) then extend the same relation to dwarf spheroidal and low-surface-brightness (LSB) galaxies.

In order to account for the above observational results the following density profile is proposed

$$
\rho(r)=\rho_{0} /\left(1+r / r_{c}\right)\left(1+r / r_{s}\right)^{2}
$$

where $\rho_{0}$ is the constant central density, $r_{c}$ is a scale factor, and $r_{s}$ is a second scale factor and is defined as $f \times r_{c}$ where $f>1$. The core radius $\left(r_{0}\right)$ is defined as that radius where the density has fallen to $\rho_{0} / 4$. (This is the definition of core radius from the Burkert (1995) profile). The observations show that $\rho_{0} r_{0}$ is a constant so that for a given assumed value of $\rho_{0}$ and $f, r_{c}$ found from a solution of the following cubic equation will satisfy this constraint.

$$
r_{c}^{3}-\left((2 / f+1) r_{0} / 3\right) r_{c}^{2}-\left(\left(2 / f+1 / f^{2}\right) r_{0}^{2} / 3\right) r_{c}-r_{0}^{3} / 3 f^{2}=0
$$

Note that with $\rho_{0} r_{0}$ given by the observations the problem contains only the two free parameters $\rho_{0}$ and $f$.

The mass enclosed within radius $r$ is given by

$M(r, a, b)=4 \pi \rho_{0}\left(1 /\left(b^{2}(a-b)(b r+1)\right)+\left((a-2 b) \ln (b r+1) /(b(a-b))^{2}+\ln (a r+1) /\left(a(a-b)^{2}\right)-1 /\left(b^{2}(a-b)\right)\right)\right.$

where $a=1 / r_{c}$ and $b=1 / r_{s}$.

The outer boundary of the model occurs when $M /\left(4 \pi r^{3} / 3\right)=200 \rho_{\text {crit }}(h=0.7$ assumed). Given that this boundary condition is the same as that assumed for standard 
CDM, the predicted baryonic Tully-Fisher relation here is also the same (i.e. $M_{b} \propto V^{3}$ ), (e.g. Steinmetz \& Navarro 1999).

In order to tie the above density distribution to a solution of the Jean's equation we use the pseudo-phase-space density $Q=\rho / \sigma_{r}^{3}$ as the second variable. This variable has interesting properties which could be relevant to the structure formation problem (Hogan \& Dalcanton, 2000; Dalcanton \& Hogan, 2001). Q was shown by Taylor and Navarro (2001) to vary with radius as $r^{-1.875}$ in the NFW model. In order to maintain this behaviour in the outer region of the above model and to keep the problem simple we assume the following form for the radial dependence of $Q$

$$
Q=Q_{0} /\left(1+\left(r / r_{Q}\right)^{1.875}\right)
$$

With the spherical Jean's equation written in terms of $\rho$ and $Q$ (Hartwick, 2008) and the above differentiable analytical expressions for both variables the solution is the run of the anisotropy parameter $\beta$ (Binney \& Tremaine, 1987) i.e.

$$
1.2 \beta=-0.6 \frac{G M_{r}}{r}\left(\frac{Q}{\rho}\right)^{2 / 3}-\frac{d \log \rho}{d \log r}+0.4 \frac{d \log Q}{d \log r}
$$

with $Q_{0}$ and $r_{Q}$ as free paramters (for fixed $\rho_{0}$ and $f$ ). By choosing $r_{Q}=r_{0}$ and varying $Q_{0}$ until $\beta \sim 0$ at $r_{200}$ solutions were found where $\beta$ rose smoothly from zero at the center to a maximum of $\sim 0.35$ followed by a not so smooth descent to zero at the outer boundary (e.g. Navarro et al. 2010, Fig. 10). Adding an extra parameter to (4) (e.g. equ'n (1) of Hartwick, 2008) results in some smoothing and changes $Q_{0}$ by $\sim \pm 20 \%$ even if $Q$ is allowed to remain at or higher than its maximum value at the outer boundary. Ultimately the observations must show the true behaviour of the run of $\beta$. $Q_{0}$ was determined by iteration using the secant method.

Under the assumption that $\rho_{0} r_{0}=120 M_{\odot} p c^{-2}$ as $f$ approaches unity a minimum mass is found for each value of $\rho_{0}$ assumed. In the case of $\rho_{0}=5.0 M_{\odot} p c^{-3}$, for example, this minimum is $\log M_{200}=7.15 M_{\odot}$ with $Q_{0}=9.8 \times 10^{-3} M_{\odot} p c^{-3}\left(\mathrm{~km} \mathrm{sec}^{-1}\right)^{-3}$. As the mass is increased at fixed central density (by increasing $f$ ) $Q_{0}$ slowly decreases. At $\rho_{0}=0.01 M_{\odot} p c^{-3}$ this minimum mass increases to $\log M_{200}=12.08 M_{\odot}$ with $Q_{0}=2.4 \times 10^{-9}$ $M_{\odot} p c^{-3}\left(\mathrm{~km} \mathrm{sec}^{-1}\right)^{-3}$. More solutions are shown in the next section where they are compared to the observations.

\section{Comparison with the Observations}

In this section we show a comparison between the model and a sample of observations. Unless otherwise stated the models were computed assuming that $\rho_{0} r_{0}=120 M_{\odot} p c^{-2}$. 


\subsection{The Fornax Dwarf Spheroidal}

Figure 1 shows the results of several analyses of the stellar kinematics of Fornax. Plotted in the $\log M-\log R$ plane are results from Strigari et al. (2007); Strigari et al. (2008); Walker et al. (2010) \& Amorisco \& Evans (2011) plotted as filled circles. The two open circles are from the work of Walker \& Penarrubia (2011) whose analysis makes use of two independent stellar populations within the galaxy. Parameters of the model shown (solid line) are $\rho_{0}=0.24 M_{\odot} p c^{-3}, f=2.0, Q_{0}=5.3 \times 10^{-6} M_{\odot} p c^{-3}\left(k m s e c^{-1}\right)^{-3} \& \log M_{200}=9.66 M_{\odot}$.

\subsection{Spiral and LSB Galaxies}

Figures $2 \& 3$ show the models superimposed on the observations of McGaugh et al. (2007) of spiral galaxies (open circles) and a sample of LSB galaxies (crosses) Kuzio de Naray et al. (2010). The spiral galaxy sample has had the mass in stars and gas removed. Figure 2 shows 4 models with density $\rho_{0}=0.3 M_{\odot} p c^{-3}$ and values of $V_{200}\left(\mathrm{kmsec}^{-1}\right)$ indicated . This is to be compared to the upper Figure 3 of McGaugh et al. (2007) where NFW models are superimposed on the same data. Note how the relation between concentration index (c) and mass cause each NFW solution to be separated from the other whereas the solutions here are approximately asymptotic about the solid black line. This line shown extended in Fig. 3 is a least squares fit to the spiral data alone and has a logarithmic slope of 0.5 (McGaugh et al. 2007). Figure 3 shows the same data superimposed by 3 models all with $M_{200}=3 \times 10^{12} M_{\odot}$ but different central densities (red, 5.0; green, $0.1 \&$ blue, $0.01 M_{\odot} p c^{-3}$ ) which illustrates the point that models with the same mass can have different dark matter distributions.

\subsection{Clusters of Galaxies}

Up to this point, all model results have been obtained by assuming $\rho_{0} r_{0}=120 M_{\odot} p c^{-2}$. This assumption appears to break down when attempting to fit the model to the observations of the galaxy cluster Abell 611 (Newman et al. 2009). These authors used a combination of weak and strong lensing along with spectroscopy of the central galaxy to obtain the dark matter distribution over a very large range in radius. In order to obtain a reasonable fit to the above observations, the model required that $\rho_{0} r_{0}=2350 \pm 200 M_{\odot} p c^{-2}$ (i.e. roughly 20 times the value assumed for the galaxies).

Recently Newman et al. (2012) have presented the results of a similar analysis for 7 massive clusters including Abell 611. The authors fit to each cluster a density profile similar to equ'n (1) but with a different parameterization. Their paper does not contain enough 
information to compute individual values of $\rho_{0} r_{0}$. However from their Fig. 3, we estimate that the central densities are $\sim 0.1-0.2 M_{\odot} p c^{-3}$ and with mean core sizes (their $r_{c}$ generally will be less than our $r_{0}$ ) of $\sim 13 k p c \rho_{0} r_{0}$ appears to be at least 10 times higher than the galaxy value. Observations of intermediate mass structures are needed to determine whether the relation between $\rho_{0} r_{0}$ and dark halo mass is continuous.

Our model fit to Abell 611 is shown in Figure 4 as the red line. The model parameters are $\rho_{0}=0.1 M_{\odot} p c^{-3}, f=9.0, Q_{0}=6.7 \times 10^{-11} M_{\odot} p c^{-3}\left(\mathrm{~km} \mathrm{sec}^{-1}\right)^{-3}$ and a mass $\log M_{200}=$ $14.57 M_{\odot}$.

\section{Summary}

The observations show that many spiral, and LSB galaxies exhibit a nearly universal relation between the underlying rotational velocity and radius at intermediate radii and a relation between the central density and core radius such that their product is a constant. A model is constructed based on these contraints and results are shown to be consistent with the observations and to differ in the inner region from predictions based on the NFW profile of CDM. Specifically, in addition to the galaxies possessing a dark matter core, the massconcentration relation appears to be absent and for central densities above a mass dependent threshold density dark halos of the same total mass can have different internal structures i.e. larger central densities and smaller core radii.

The model was also fit to the observations of the galaxy cluster Abell 611. It was found that the central density-core radius relation had to be $\sim 20$ times higher than that found for the galaxy sample. A recent study of the density profile of 7 massive clusters by Newman et al. (2012) also suggests a significantly higher value. Additional observations are required to determine if $\rho_{0} r_{0}$ varies continuously between the masses of the dark matter halos of the galaxies and those of the galaxy clusters.

The model provides an empirically-motivated cored template which may be useful for disentangling the relative contributions of dark matter and baryons in other types of galaxies such as the ellipticals.

Should the observational constraints which define the model be shown to be compatible with other galaxy types (e.g. the ellipticals) then accounting for them will strengthen the need for major modifications to the standard CDM picture.

One way to modify CDM is to invoke the idea that the dark matter particles are self interacting (e.g. Spergel \& Steinhardt 2000). If the mean free path of the particles is of 
the order of the core size $r_{0}$, then the scattering coefficient will be $\sim\left(\rho_{0} r_{0}\right)^{-1}$. In the case of Abell 611 this quantity in cgs units is $2.0 \pm 0.2 \mathrm{~cm}^{2} \mathrm{gm}^{-1}$. However to be consistent with the value for the galaxies, $\sim 40.0 \mathrm{~cm}^{2} \mathrm{gm}^{-1}$, the scattering coefficient could be energy dependent given the difference in mass between the galaxies and the clusters. Such an energy dependent scattering coefficient has been investigated by Loeb \& Weiner (2011). A further consideration is that if the coefficient is sufficiently large the halo is subject to core collapse within a Hubble time (e.g. Burkert 2000). Clearly, many uncertainties with this scheme remain.

The self interacting dark mattter (SIDM) picture mentioned above is but one of several which have been proposed to explain the presence of cores in dark matter halos. An up-todate list is discussed in the paper by Newman et al. (2012). The challenge for any theory is to predict the observed relation between $\rho_{0}$ and $r_{0}$. The continuing observational program of separating the distribution baryons and dark matter on all scales and for all morphological structures should eventually lead to the correct scenario.

The author wishes to thank Andrew Newman and Richard Ellis for kindly providing the data from Newman et al. (2009) on the galaxy cluster Abell 611 in the form shown in Fig 4. He also gratefully acknowledges support for this work from a discovery grant from NSERC (Canada).

\section{REFERENCES}

Amorisco, N. C., \& Evans, N. W. 2011, MNRAS, 411, 2118

Binney, J., \& Tremaine, S. 1987, Galactic Dynamics, (Princeton: Princeton University Press)

Burkert, A. 1995, ApJ, 447, L25

Burkert, A. 2000, ApJ, 534, L143

Dalcanton, J.J., \& Hogan, C.J. 2001, ApJ, 561, 35

Donato, F., Gentile, G., Salucci, P., Frigerio Martins, C., Wilkinson, M. I., Gilmore, G., Grebel, E. K., Koch, A., \& Wyse, R. 2009, MNRAS, 397, 1169

Hartwick, F. D. A. 2008, ApJ, 674, 220

Hogan, C. J. \& Dalcanton, J. J. 2000, Physical Review D, 62, 063511

Kormendy, J., \& Freeman, K. C. 2004, IAU Symposium 220, 377

Kuzio de Naray, Rachel, Martinez, Gregory D., Bullock, James S., Kaplinghat, Manoj 2010, ApJ, 710, L161 
Loeb, Abraham, \& Weiner, Neal 2011, Physical Review Letters, 106, 171302

McGaugh, S. S., de Blok, W. J. G., Schombert, J. M., Kuzio de Naray, R., Kim, J. H. 2007, ApJ, 659,149

Navarro, Julio F., Frenk, Carlos S., \& White, Simon D. M. 1997, ApJ, 490, 493

Navarro, Julio F., Ludlow, Aaron, Springel, Volker, Wang, Jie, Vogelsberger, Mark, White, Simon D. M., Jenkins, Adrian, Frenk, Carlos S., \& Helmi, Amina 2010, MNRAS, 402, 21

Newman, Andrew B., Treu, Tommaso, Ellis, Richard S., Sand, David J., Richard, Johan, Marshall, Philip J., Capak, Peter, \& Miyazaki, Satoshi 2009, ApJ, 706, 1078

Newman,Andrew B., Treu, Tommaso, Ellis, Richard S., \& Sand, David J. 2012, ApJ, in press, astro-ph:1209.1392v1

Salucci, Paolo, Wilkinson, Mark I., Walker, Matthew G., Gilmore, Gerard F., Grebel, Eva K., Koch, Andreas, Frigerio Martins, Christiane, \& Wyse, Rosemary F. G. 2012, MNRAS, 420, 2034

Spergel, D. N. \& Steinhardt, P. J. 2000, Physical Review Letters, 84, 3760

Steinmetz, M. \& Navarro, J. F. 1999, ApJ, 513, 555

Strigari, Louis E., Bullock, James S., Kaplinghat, Manoj, Diemand, Juerg, Kuhlen, Michael, \& Madau, Piero 2007, ApJ, 669, 676

Strigari, Louis E., Bullock, James S., Kaplinghat, Manoj, Simon, Joshua D., Geha, Marla, Willman, Beth, \& Walker, Matthew G. 2008, Nature, 454, 1096

Taylor, James E., \& Navarro, Julio F. 2001, ApJ, 563, 483

Walker, Matthew G., McGaugh, Stacy S., Mateo, Mario, Olszewski, Edward W., Kuzio de Naray, Rachel 2010, ApJ, 717, 187

Walker, Matthew G., \& Pearrubia, J. 2011, ApJ, 742, 20

Walker, Matthew G., Mateo, Mario, Olszewski, Edward W., Pearrubia, Jorge, Wyn Evans, N., \& Gilmore, G. 2010, ApJ, 710, 886 


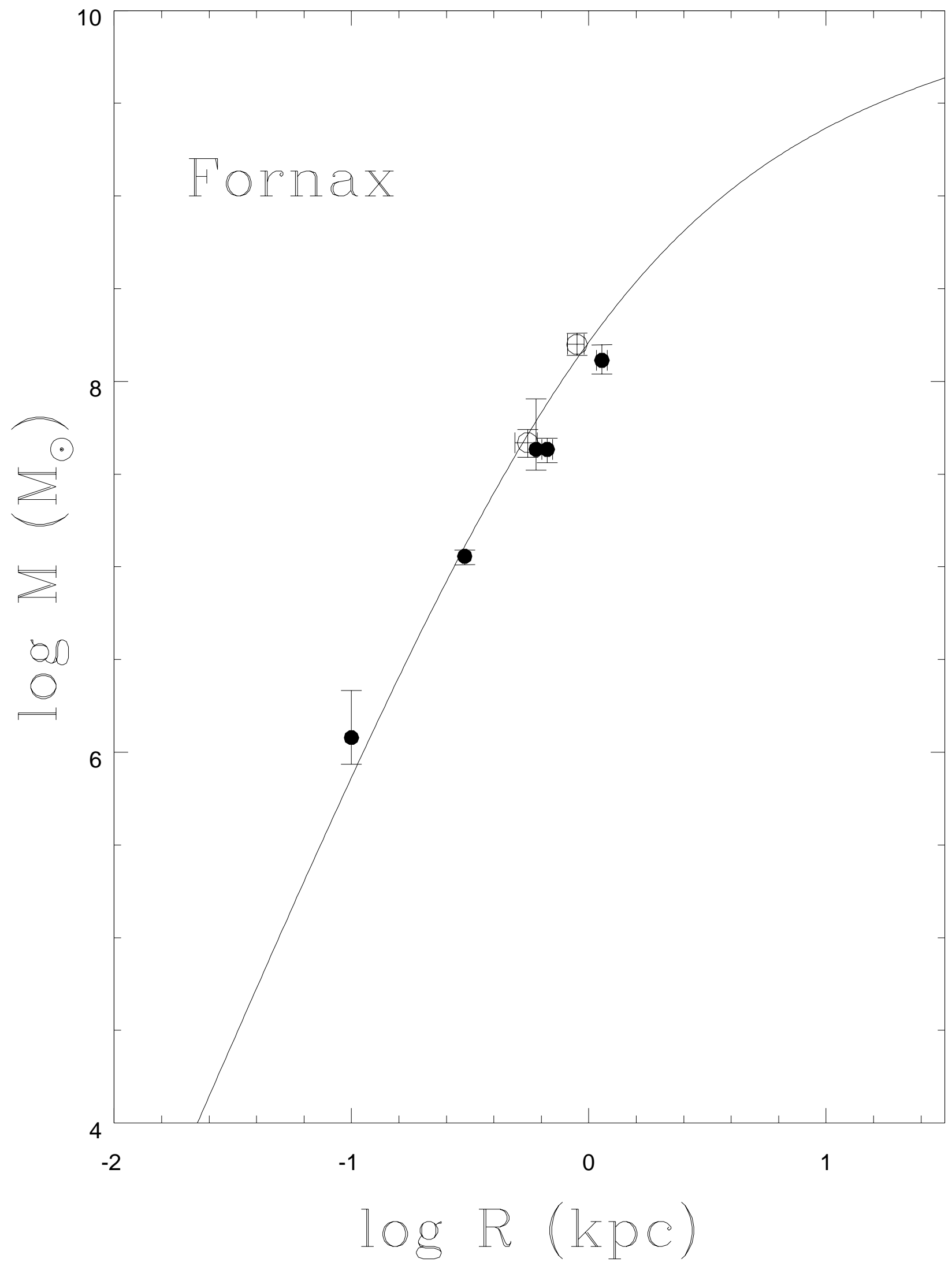

Fig. 1.- A model with parameters $\rho_{0}=0.24, f=2, Q_{0}=5.3 \times 10^{-6} \& \log M_{200}=9.66 M_{\odot}$ fit to the modelled stellar kinematical data cited in the text for the Fornax Dwarf Spheroidal Galaxy 


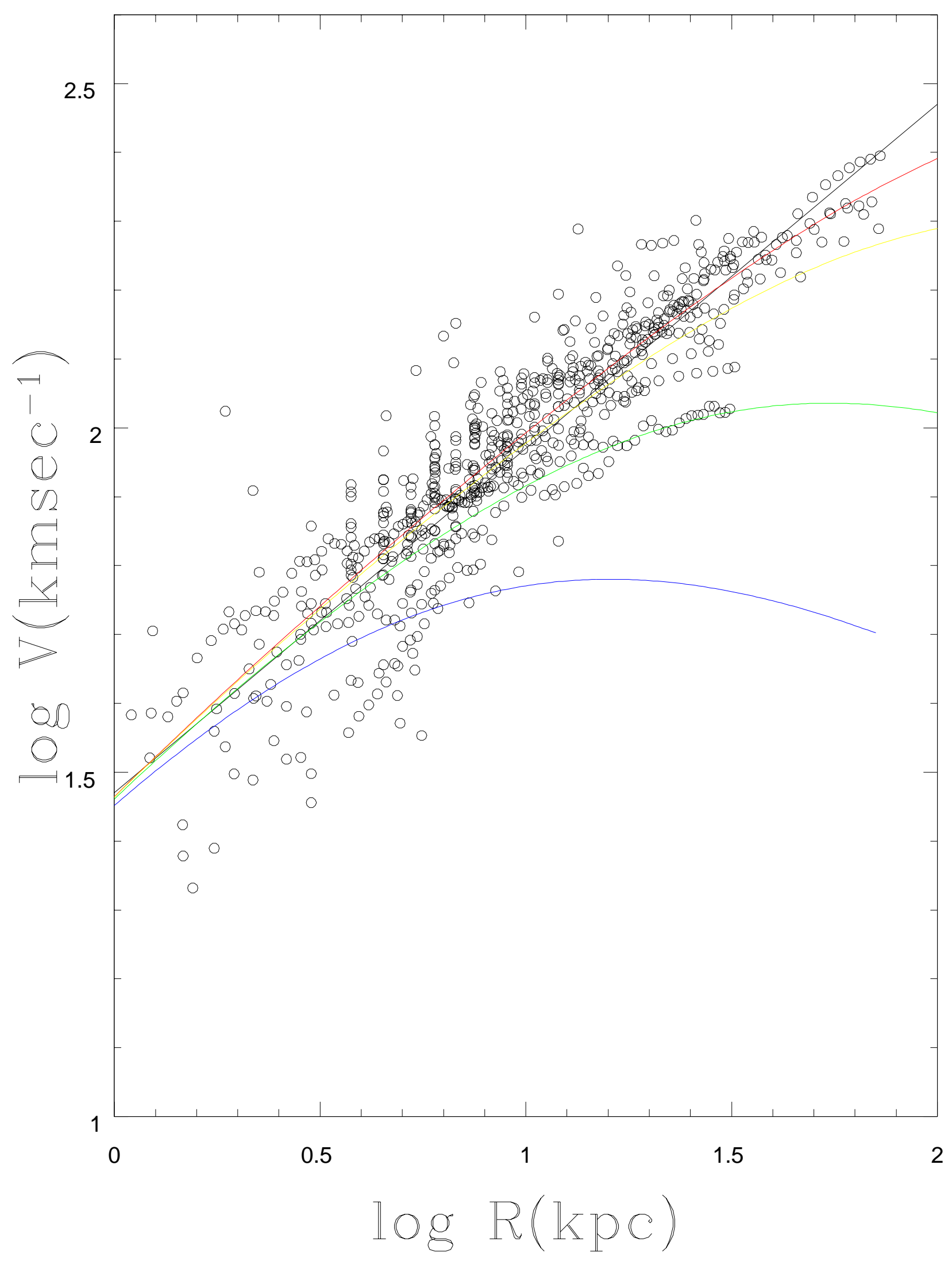

Fig. 2.- Four models superimposed on the data from fig 3 of McGaugh et al. (2007). All models have $\rho_{0}=0.3$ but with $V_{200}=300, Q_{0}=7.1 \times 10^{-7}(\mathrm{red}) ; V_{200}=200, Q_{0}=1.1 \times 10^{-6}$ (yellow); $V_{200}=100, Q_{0}=2.2 \times 10^{-6}($ green $) ; \& V_{200}=50, Q_{0}=4.3 \times 10^{-6}$ (blue) 


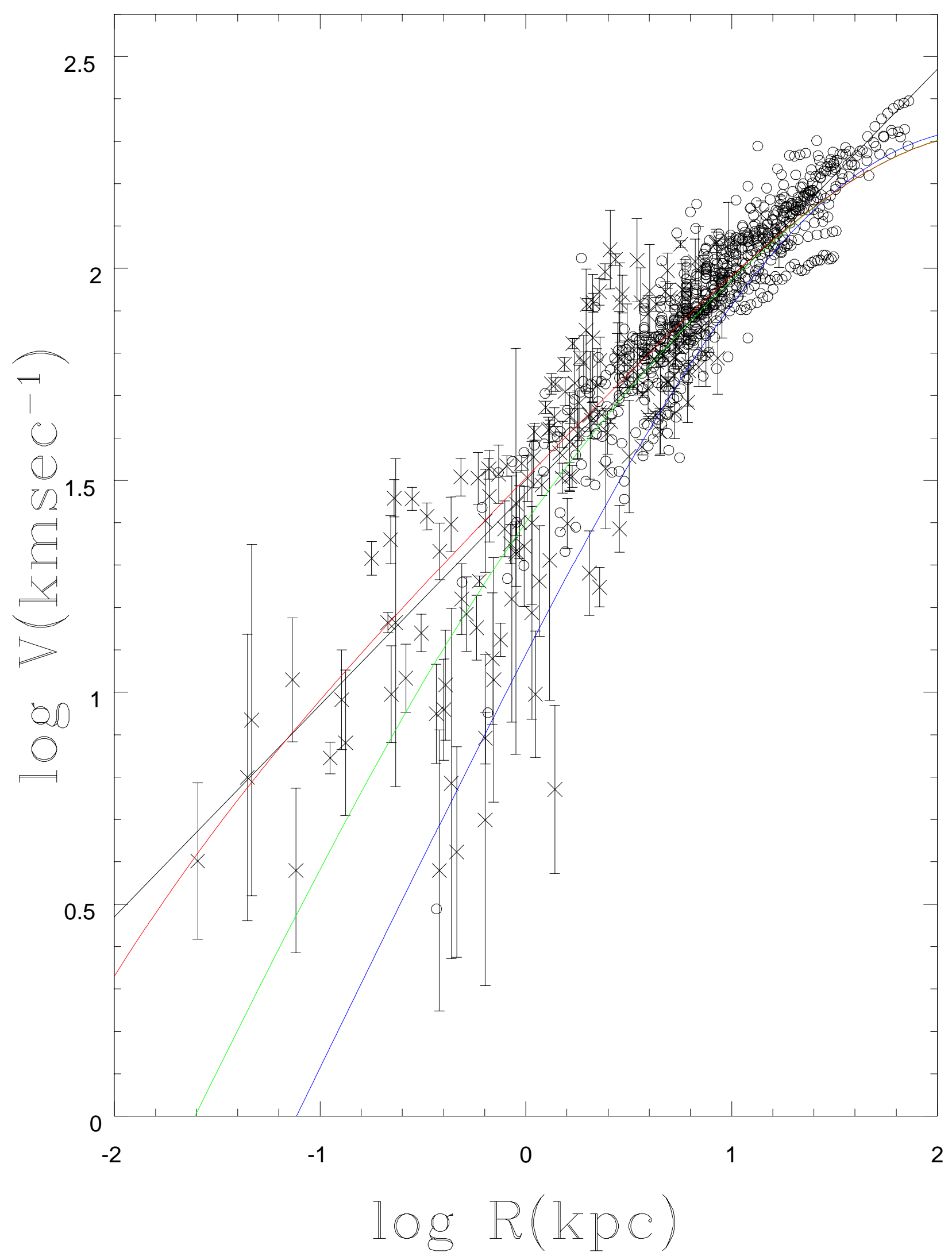

Fig. 3.- Three models superimposed on the data from fig 3 of McGaugh et al. (2007) extended to include data for LSB galaxies from Kuzio de Naray et al. (2010) shown as crosses. All three models have the same $M_{200}=3 \times 10^{12} M_{\odot}$ but different central densities; 


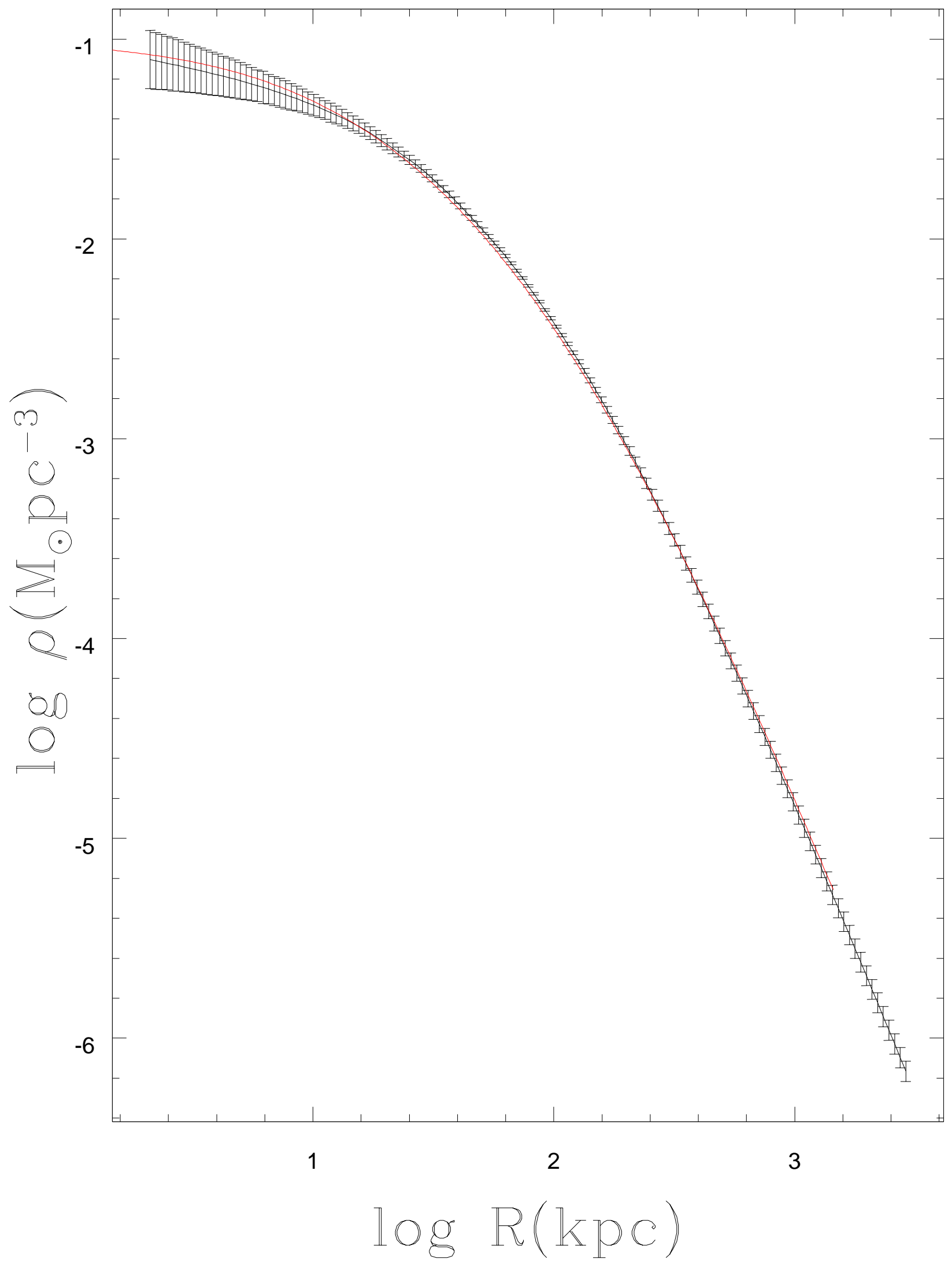

Fig. 4.- A model (red line) with $\rho_{0} r_{0}=2350 M_{\odot} p c^{-2}$ fit to the observations of Abell 611 (Newman et al. 2009). The parameters are $\rho_{0}=0.1, f=9, Q_{0}=6.7 \times 10^{-11} \& \log M_{200}=$ $14.57 \mathrm{M}$ 